dielectric anisotropic LCs, and a test NIVAGH-LC cell was manufactured using two indium-tin oxide (ITO) substrates separated by $5 \mu \mathrm{m}$ bead spacers. The test cell exhibited a low threshold voltage of $\sim 2.1 \mathrm{~V}_{\mathrm{rms}}$ and a high reflectance of $\sim 59 \%$ in the voltage-off state.

The researchers reported that optimal vertical alignment depends on the magnitude differences between the POSS-LC and LC-LC interactions. The researchers said that the NIVA phenomenon is due to the adsorption of POSS nanoparticles on the inner surface of the ITO substrates, which lowers the surface tension, thereby inducing homeotropic alignment.

The NIVA technique is advantageous because it can be applied at room temperature and is a relatively simple process for preparing GH-LCDs. The resulting NIVA-GH-LC cells are favorable for lowcost applications, such as price tags and timepieces, which require plastic substrates and low power consumption. The researchers demonstrated this by fabricating a flexible timepiece with $1 \mathrm{wt} \%$ dye and $8.5 \mu \mathrm{m}$ spacers. The next steps are to examine the electro-optical properties and reliability of the plastic NIVAGH-LC under mechanical bending, said the researchers.

SAMESHA R. BARNES

\section{Terbium Phosphor Exhibits High Spectral Purity in the Green}

The color purity of the emission spectrum of terbium-doped crystals excited at wavelengths shorter than $380 \mathrm{~nm}$ depends on $\mathrm{Tb}^{3+}$ concentration. At low $\mathrm{Tb}^{3+}$ concentrations, the emission is dominated by the ${ }^{5} \mathrm{D}_{3}-{ }^{7} \mathrm{~F}_{\mathrm{J}}(\mathrm{J}=0-6)$ transitions with several lines in the blue region. With increasing $\mathrm{Tb}^{3+}$ concentration, the ${ }^{5} \mathrm{D}_{4}-{ }^{7} \mathrm{~F}_{\mathrm{J}}$ transitions become dominant, generating blue, green, yellow, and red lines. However, M. Gusowski and W. RybaRomanowski, from the Polish Academy of Sciences in Wroclaw, Poland, reported in the August issue of Optics Letters (DOI: 10.1364/OL.33.001786; p. 1786 ) the development of $\mathrm{K}_{3} \mathrm{YF}_{6}: \mathrm{Tb}^{3+}$-a new phosphor in which, in contrast to other terbium-doped materials reported until now, the emission is independent of the $\mathrm{Tb}^{3+}$ concentration and most of the intensity of the green emission was confined in an extremely narrow spectral bandwidth.

The researchers prepared a series of polycrystalline samples $\mathrm{K}_{3} \mathrm{Y}_{1-x} \mathrm{~Tb}_{x} \mathrm{~F}_{6}(x=$ $0.03,0.1,0.2$, and 1 ) by heating stoichiometric mixtures of $\mathrm{KF}_{3}, \mathrm{YF}_{3}$, and $\mathrm{TbF}_{3}$ at $800^{\circ} \mathrm{C}$ in graphite crucibles in Ar atmosphere. They obtained monoclinic crystals with space group $\mathrm{P} 2_{1} / \mathrm{n}$. Yttrium or terbium ions were sixfold-coordinated by fluorine ions and occupied strongly distorted octahedrons with centrosymmetric $C_{i}$ local symmetry. The emission spectra were recorded with a fluorometer system consisting of a $150 \mathrm{~W}$ xenon lamp coupled to an excitation monochromator and a scanning grating monochromator equipped with a photomultiplier. Excitation at a sample temperature of $8 \mathrm{~K}$ by $375 \mathrm{~nm}$ light yielded a narrow-band emission at around $545 \mathrm{~nm}$, formed by three line components peaking at $540.77 \mathrm{~nm}, 541.18 \mathrm{~nm}$, and $541.74 \mathrm{~nm}$, related to transitions from the lowest crystal field component of the ${ }^{5} \mathrm{D}_{4}$ multiplet to crystal field components of the terminal ${ }^{7} \mathrm{~F}_{5}$ multiplet.

The researchers considered that, although 11 field components were predicted for the ${ }^{7} \mathrm{~F}_{5}$ multiplet of $\mathrm{Tb}^{3+}$ in $\mathrm{C}_{\mathrm{i}}$ symmetry, only three of them were involved in the emission. The researchers think that pure electric-dipole transitions were forbidden by the centrosymmetric site positions of $\mathrm{Tb}^{3+}$ in $\mathrm{K}_{3} \mathrm{YF}_{3}$, and only the ${ }^{5} \mathrm{D}_{4}-{ }^{-7} \mathrm{~F}_{5}$ magnetic-dipole transition remained. When the temperature was increased, an additional line with two distinct maxima grew at longer wavelengths. The long lifetime at room temperature of the ${ }^{5} \mathrm{D}_{4}$ manifold (14 ms), together with the negligible intensities of other transitions, demonstrate to the researchers that the electron-phonon coupling in this phosphor was low.

The researchers conclude that this phosphor can be efficiently excited in the UV-VUV region through the intense $\mathrm{f}-\mathrm{d}$ transitions of $\mathrm{Tb}^{3+}$ and that it can be of interest for applications requiring high spectral purity in the green region of the electromagnetic spectrum.

JOAN J. CARVAJAL

\section{Railed Microfluidic Channels Enable Precise Microstructure Assembly}

Robotic assembly of small parts $(<200$ $\mu \mathrm{m}$ in size) is difficult because of the precision of the control requirements. Fluidic self-assembly is an alternative approach, which has suffered from low yield because

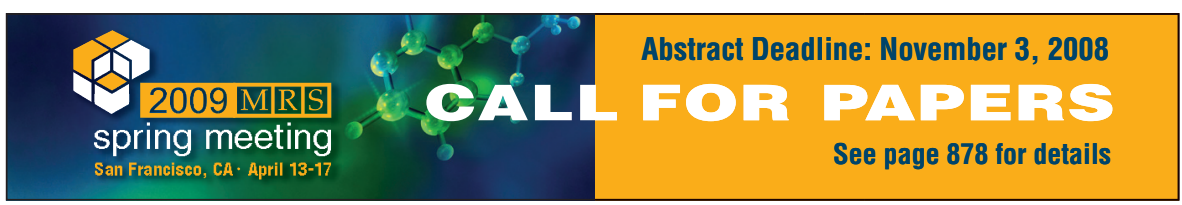

of the probabilistic nature of self-assembly processes. Recently, a simplified manufacturing design was created that has the ability to integrate dissimilar parts into threedimensional geometries while replicating them in parallel. S.E. Chung, W. Park, and their colleagues at Seoul National University in the School of Electrical Engineering and Computer Science have developed this self-assembly technique using railed microfluidic channels to assemble accurate complex structures.

As described in the July issue of Nature Materials (DOI: 10.1038/nmat2208; p. 581), the researchers constructed a microfluidic device out of polydimethylsiloxane (PDMS). Unlike other microfluidic mechanisms, the microchannels of this device have raised structures, called rails, which are grooved on the top surface of the channel. These rails act as a guide for microtrains that contain micro- and nanosize parts that can be photopolymerized in situ, transported along the rail-line by fluid flow, and assembled at the end of the rail. The benefit of this technique is that many intricate components of varying materials can be assembled at a high yield.

To show the applicability of this technique, the researchers assembled an array of living cells (to demonstrate the utility of this approach to biomedical applications) and also an array of externally fabricated silicon microchips (to demonstrate the utility of this approach to microelectronic or optoelectronic applications). The living cell matrix, a $3 \times 3$ hydrogel, was produced by maneuvering two types of living cells (one type transfected by a red fluorescent protein and the other by a green fluorescent protein) into a checkerboard pattern, eliminating multiple lithography steps. The researchers said that this process could be easily adapted to arrays of cells of multiple cell types.

To fabricate the array of microchips, a set of $100 \mu \mathrm{m} \times 100 \mu \mathrm{m}$ silicon chips was propelled through the fluid channel in a polyethylene glycol diacrylate solution. At the end of the rail, a single-step lithography process created an array pattern on the chip with desired spacing. The researchers said that this approach may have commercial applications "for LED [light-emitting diode]-based back-light units or LED lighting packaging where a large number of small LED chips need to be placed in a larger substrate, such as a glass plate or a silicon wafer." From this research, the investigators said that their system may produce nearly zero assembly error in almost any configuration and that railed assembly may be the preferred method of self-assembly of the future.

TARA WASHINGTON 